\title{
Monitoring the acute phase response
}

\author{
Alternative tests to measuring erythrocyte sedimentation rate
}

Local damage to tissue invokes a systemic response that includes fever, a neutrophil leucocytosis, and an increase in the concentration of plasma proteins. This inflammatory (acute phase) response is mediated by cytokines such as interleukin $1^{1}$ that are released from macrophages at the site of tissue damage. The initiating pathological process may be immunological, infective, ischaemic, malignant, or traumatic - so that methods for monitoring the acute phase response have wide applications.

Since 1921 measuring the erythrocyte sedimentation rate has been the most popular method for assessing the protein component of this response. ${ }^{23}$ In 1977 the International Committee for Standardization in Haematology described a standardised version of the Westergren technique as the recommended method for measuring the erythrocyte sedimentation rate. ${ }^{4}$ Advocates of the test cite its usefulness and reliability in monitoring the progress of inflammatory disease; moreover, it may be used as a non-specific screening test for disease. Critics have suggested that measurement of the erythrocyte sedimentation rate should be abandoned because it is strongly influenced by anaemia, which cannot be corrected for and thus confuses the interpretation. Quality control of this manual procedure is also unsatisfactory.

Despite serious questions about the validity of measuring the erythrocyte sedimentation rate the test is still widely used because it is cheap, easy to perform, and does not require a power supply, investment in capital equipment, or much technical skill. It is especially attractive for small laboratories, primary health clinics, and countries with limited resources.

The expert panel on blood rheology of the International Committee for Standardization in Haematology has recently prepared guidelines on selecting laboratory tests for monitoring the acute phase response. ${ }^{5}$ Quantitative assay of $\mathrm{C}$ reactive protein concentration is recommended as the best test when changes in the acute phase response occur within 24 hours. An increase in plasma concentration of $C$ reactive protein may be detected within six to 10 hours after tissue damage, and as the inflammatory response subsides the plasma concentration falls with a half time of 48 hours. Thus the test will monitor the onset of inflammation or its response to treatment. Semiquantitative and qualitative assays for $\mathrm{C}$ reactive protein concentration are not recommended as they are not sufficiently sensitive to monitor short term changes.

An increase in the erythrocyte sedimentation rate reflects a rise in the plasma concentration of proteins, such as fibrinogen and some immunoglobulins, that are of large molecular size and aggregate red cells to form rouleaux. An increase in plasma concentration of fibrinogen may not be seen until 24-48 hours after inflammation begins, and the concentration subsequently decreases with a half time of four to six days. Thus measuring the erythrocyte sedimentation rate is not valuable for monitoring rapid changes in the onset or resolution of the acute phase response.

Plasma proteins that increase the erythrocyte sedimentation rate also increase plasma viscosity, which might be measured instead of the erythrocyte sedimentation rate. ${ }^{6}$ Measuring the erythrocyte sedimentation rate takes at least one hour and should be performed within two hours after venepuncture whereas plasma viscosity may be measured in minutes and on stored samples. The result is independent of anaemia and gender and is largely independent of age except in newborn infants and very old people. Laboratory calibration and quality control are also much easier. Now that manufacturers are beginning to develop automated viscometers for hospital laboratories ${ }^{7}$ measurement of plasma viscosity instead of erythrocyte sedimentation rate is likely to increase.

The erythrocyte sedimentation rate and plasma viscosity are sensitive to the combined effect of several proteins and are therefore useful for monitoring the more complex hyperproteinaemia of the acute phase response after 24 hours, including the protein changes of chronic disease. Neither is valuable for monitoring the short term changes of disease. Indeed, lack of sensitivity of these tests to short term changes may be of positive value in assessing the long term changes of chronic disease. The guidelines from the international committee recommend that tests for monitoring short term and long term changes in acute phase proteins are complementary and should be used for different clinical purposes. Clinicians and pathologists should be aware of these guidelines so that the best tests may be selected to match local needs and resources. The ability to measure $C$ reactive protein concentration and plasma viscosity within minutes and also on stored specimens has implications that range from monitoring newborn infants ${ }^{8}$ to providing rheumatologists with a rapid and quantitative measurement of the acute phase response for outpatient clinics. It is unlikely that 
measurement of the erythrocyte sedimentation rate will be abandoned, but its limitations should be recognised.

Professor of Haematology,

JOHN STUART

Medical School,

University of Birmingham,

Birmingham B15 2TJ

Consultant Haematologist,

S M LEWIS

Royal Postgraduate Medical School,

London W12 0HS
1 Dinarello CA. Interleukin-1 and the pathogenesis of the acute-phase response. $N$ Engl F Med 1984;311:1413-8.

2 Fåhraeus R. The suspension-stability of the blood. Acta Med Scand 1921;55:1-228.

3 Westergren $\mathrm{A}$. Studies of the suspension stability of the blood in pulmonary tuberculosis. Acta Med Scand 1921;54:247-82.

4 International Committee for Standardization in Haematology. Recommendation for measurement erythrocyte sedimentation rate of human blood. Am f Clin Pathol 1977;68:505-7.

5 International Committee for Standardization in Haematology (Expert Panel on Blood Rheology). Guidelines on selection of laboratory tests for monitoring the acute phase response. $\mathcal{F}$ Clin Pathol Guidelines on selectic $1988: 41: 1203-12$.

6 Harkness J. The viscosity of human blood plasma; its measurement in health and disease. Biorheology 1971;8:171-93.

7 Cooke BM, Stuart J. Automated measurement of plasma viscosity by capillary viseometer. $\mathcal{f}$ Clin Pathol 1988;41:1213-6.

8 Stuart J, Whicher JT. Tests for detecting and monitoring the acute phase response. Arch Dis Child 1988;63:115-7.

\title{
I don't want you to see a psychiatrist
}

\author{
Non-psychiatrists provide most mental health care
}

Twenty five years ago Kessel asked who ought to receive psychiatric care in Britain. ${ }^{1} \mathrm{He}$ argued that psychiatrists should welcome increasing public demand for their services and that general practitioners should be encouraged to refer patients freely to psychiatrists "when they want advice." As we now know most acutely ill patients with psychotic disorders see a psychiatrist. The rest of those with psychiatric problems-about $95 \%$ of the total ${ }^{2}$-are treated by general practitioners, usually alone but sometimes together with various non-medical providers of mental health care. General practitioners are turning increasingly to nurses, social workers, clinical psychologists, and counsellors rather than to psychiatrists for help for their patients with mental health problems.

General practitioners are the largest group referring patients to community psychiatric nurses. ${ }^{3}$ Community psychiatric nurses working in health centres are most commonly asked to help with patients with mood disorders, but they also treat patients with neurotic and psychotic disorders. ${ }^{4}$ Two randomised controlled trials have shown clinical and economic benefits from nurses treating patients with neurotic disorders in the community. Firstly, community psychiatric nursing was compared with routine outpatient psychiatric follow up over 18 months. Community psychiatric nursing resulted in an appreciable reduction in outpatients' contacts with psychiatrists and other staff, more discharges, and a small increase in contact with general practitioners for prescribing. ${ }^{56}$ Secondly, neurotic patients (mainly those with phobic and obsessive-compulsive disorders) had a better outcome one year after receiving behavioural psychotherapy from a nurse therapist than after routine treatment from a general practitioner. ${ }^{78}$ Practice nurses already provide much emotional support to patients with physical and psychiatric illness, though this is largely unrecorded. In addition, health visitors are important in identifying and treating emotional problems in women who have recently given birth. ${ }^{9}$

Two randomised controlled trials have shown the effectiveness of social work for depressed patients. In one study women suffering from acute or acute on chronic depression were referred to a social worker attached to a general practice or for routine treatment by their general practitioners. Women with acute on chronic depression and large marital difficulties benefited from treatment by social workers. ${ }^{10}{ }^{11}$ In the other study depressed patients were allocated to individual cognitive therapy, group cognitive therapy, or a waiting list control group. ${ }^{12}$ Those who had cognitive therapy from a social worker did significantly better up to one year than those on the waiting list, but there was no significant difference between patients treated with group or individual cognitive therapy.

General practitioners refer to clinical psychologists patients with difficulties ranging from anxiety, phobia, depression, and psychosomatic conditions to habit disorders, behavioural, personality, interpersonal, social, marital, sexual, educational, and occupational problems, and cognitive impairment. Patients show high satisfaction with behavioural treatment, ${ }^{13}$ and they have a third to a half fewer consultations for advice or prescriptions for psychotropic drugs in the year after psychological intervention. ${ }^{14}$ is Such benefits have been confirmed up to one year in a randomised controlled clinical and economic evaluation of a behaviourally oriented clinical psychology service in a health centre. ${ }^{16}$ Contact with a psychologist may have effects on referred patients and their families over the longer term, with decreases at three years in the number of prescriptions for psychotropic drugs for their children. ${ }^{17}$ Advantages have also been shown for specific psychological treatments in patients with depression and anxiety. Two controlled clinical trials have produced favourable early results for psychologists using cognitive therapy combined with antidepressants in treating depressive disorders. ${ }^{1819}$ Group psychological treatment for anxiety has been compared with individual treatment: individual treatment was more effective in reducing anxiety and service demands were considerably reduced by group treatment. ${ }^{20}$

A growing number of counsellors are being recruited into primary care. Individual, family, group, and marital counselling are used, and the counsellor's main aim is to offer the patient support and insight. Patients are also given the chance to learn new skills, such as relaxation, and vocational and educational guidance may be given. Several clinical accounts show the impact of counselling in general practice-for example, on subjective feelings of patients and general practitioners and on reductions in the number of consultations and prescriptions for psychotropic drugs. ${ }^{21-24}$ People with marital difficulties are more likely to contact their general practitioners for help than any other social service, and several attachments of marriage guidance counsellors to general practice have been set up to encourage doctors to refer patients directly. ${ }^{25}$ These attachments seem to work well, but the experience is limited to self selected and atypical practices.

We have done a meta-analysis ${ }^{26}$ on 11 British studies $^{810121316}$ $192327-30$ of specialist mental health treatment in general practice. In each study the outcome of treatment by a specialist mental health professional located in general practice was compared quantitatively with the outcome of the usual 